\title{
Resíduos sólidos urbanos de poda de gramíneas como fonte alternativa de energia: um estudo de caso na cidade de Rosana
}

\author{
Urban solid waste from grass pruning as an alternative energy source: a case study in the city of
} Rosana

Residuos sólidos urbanos de la poda de césped como fuente de energía alternativa: un estudio de caso en la ciudad de Rosana

\author{
Caio Mendonça \\ ORCID: https://orcid.org/0000-0002-2891-7836 \\ Universidade Estadual Paulista, Brasil \\ E-mail: caiobm97@gmail.com \\ João Pedro Jenson de Oliveira \\ ORCID: https://orcid.org/0000-0003-1355-2886 \\ Universidade Estadual Paulista, Brasil \\ E-mail: joaojenson1@gmail.com \\ Carlos Toshiyuki Hiranobe \\ ORCID: https://orcid.org/0000-0002-5182-2018 \\ Universidade Estadual Paulista, Brasil \\ E-mail: carlost.hiranobe@unesp.br \\ Renivaldo José dos Santos \\ ORCID: https://orcid.org/0000-0002-0079-6876 \\ Universidade Estadual Paulista, Brasil \\ E-mail: renivaldo.santos@unesp.br \\ Leonardo Lataro Paim \\ ORCID: https://orcid.org/0000-0002-5113-6376 \\ Universidade Estadual Paulista, Brasil \\ E-mail: leonardo.paim@unesp.br
}

\begin{abstract}
Resumo
O objetivo deste trabalho foi estudar os resíduos sólidos urbanos de poda de gramíneas (RSPG) do Distrito de Primavera, no município de Rosana-SP através de métodos gravimétricos e calorimétrico para avaliar a viabilidade de utilização como fonte de energia em processos de combustão direta. As amostras de RSPG foram coletadas, lavadas, secas ao ar por $24 \mathrm{~h}$, fracionadas em moinho de facas e selecionadas em peneira granulométrica antes das análises gravimétricas e calorimétrica. Foram realizadas análises de umidade, sólidos totais, extrativos aquoso e alcoólico, cinzas, inorgânicos estruturais e não-estruturais, inorgânicos totais e poder calorífico superior (PCS). O processo de extração aquosa e alcoólica foram realizados em banho ultrassônico e apresentaram os valores de 14,32 \pm 0,28 \% para os extrativos aquosos e 6,65 \pm 0,40 \% para os extrativos alcoólicos. As amostras apresentaram valores de inorgânicos estruturais e inorgânicos não estruturais de $2,97 \pm 0,17 \%$ e $1,26 \pm 0,12 \%$, respectivamente. O RSPG apresentou ser alternativa como combustível sólido ao apresentar um valor de PCS de $17,59 \pm 0,43 \mathrm{MJ} \mathrm{kg}^{-1}$ que é similar a outros resíduos sólidos.
\end{abstract}

Palavras-chave: Biomassa; Resíduo sólido urbano; PCS; Bioenergia; Potencial energético.

\begin{abstract}
The aim of this work was to study urban solid waste from grass pruning (RSPG) at District of Primavera, in RosanaSP, using gravimetric and calorimetric methods to evaluate the feasibility of using it as an energy source in direct combustion processes. RSPG samples were collected, washed, dried in air for $24 \mathrm{~h}$, fractionated in a knife mill and selected in a granulometric sieve before gravimetric and calorimetric analyses. Analyzes of moisture, total solids, aqueous and alcoholic extractives, ash, structural and non-structural inorganics, total inorganics, and higher heating value (HHV) were carried out. The aqueous and alcoholic extraction processes were carried out in an ultrasonic bath and presented values of $14.32 \pm 0.28 \%$ for aqueous extractives and $6.65 \pm 0.40 \%$ for alcoholic extracts. The samples showed values of structural inorganics and non-structural inorganics of $2.97 \pm 0.17 \%$ and $1.26 \pm 0.12 \%$, respectively. RSPG proved to be an alternative as solid fuel by presenting a HHV value of $17.59 \pm 0.43 \mathrm{MJ} \mathrm{kg}^{-1}$ which is like other solid wastes.
\end{abstract}

Keywords: Biomass; Urban solid waste; HHV; Bioenergy; Energy potential. 


\section{Resumen}

El objetivo de este trabajo fue estudiar los residuos sólidos urbanos de la poda de gramíneas (RSPG) en el Distrito de Primavera, en Rosana-SP, utilizando métodos gravimétricos y calorimétricos para evaluar la factibilidad de utilizarlos como fuente de energía en procesos de combustión directa. Las muestras de RSPG se recolectaron, lavaron, secaron al aire durante $24 \mathrm{~h}$, se fraccionaron en un molino de cuchillas y se seleccionaron en un tamiz granulométrico antes de los análisis gravimétricos y calorimétricos. Se realizaron análisis de humedad, sólidos totales, extractos acuosos y alcohólicos, cenizas, inorgánicos estructurales y no estructurales, inorgánicos totales y poder calorífico superior (PCS). Los procesos de extracción acuosa y alcohólica se realizaron en baño ultrasónico y presentaron valores de $14,32 \pm 0,28 \%$ para extractos acuosos y $6,65 \pm 0,40 \%$ para extractos alcohólicos. Las muestras presentaron valores de inorgánicos estructurales e inorgánicos no estructurales de $2.97 \pm 0.17 \%$ y $1.26 \pm 0.12 \%$, respectivamente. El RSPG demostró ser una alternativa como combustible sólido al presentar un valor de PCS de 17,59 $\pm 0,43 \mathrm{MJ}^{\mathrm{kg}} \mathrm{g}^{-1}$ que es similar a otros residuos sólidos.

Palabras clave: Biomasa; Residuos sólidos urbanos; PCS; Bioenergía; Potencial energético.

\section{Introdução}

Recentemente temos vivenciado situações energéticas adversas no Brasil, onde devido ao desmatamento contínuo para alimentar a indústria agropecuária, uma crise hídrica se instaurou durante o período de 2020 e 2021 . Com a baixa precipitação, as usinas hidrelétricas têm tido dificuldade para manter seus reservatórios com o volume para o funcionamento ideal (Haidar, 2021; Miranda et al., 2013).

Hoje o Brasil possui uma matriz energética amplamente diversificada, contudo, muito dependente da energia hidráulica provinda de usinas hidrelétricas que representam cerca de 65,2\% da matriz (Empresa de Pesquisa Energética, 2021). É necessário incentivar a produção de energia limpa e de fontes renováveis provindas de outras matérias-primas, como a biomassa, que hoje representa apenas 9,1\% da matriz energética (Empresa de Pesquisa Energética, 2021). Essa parcela da matriz representada pela biomassa poderia ser muito maior, pois o país é um território amplo e vasto de resíduos verdes que são "desperdiçadas", como as folhas das árvores e as podas de grama que são materiais orgânicos e que podem ser usados na geração de energia termoelétrica assim como a cana-de-açúcar (Hiloidhari \& Baruah, 2011).

Com isso, diversos estudos têm surgido acerca de resíduos potencialmente utilizáveis para a geração de energia termoelétrica. A maioria desses resíduos são matérias orgânicas como a palha, folhas, gramíneas, tocos, galhos, bagaço da cana-de-açúcar, casca de arroz e resíduos das madeireiras (de Sousa et al., 2021; Evaristo et al., 2020; Gomes et al., 2020; La Picirelli de Souza et al., 2021).

É notável que a utilização desses subprodutos pode acarretar um excelente crescimento econômico, gerando benefícios nos âmbitos sociais, empresariais e governamentais, e ainda promover uma melhora na qualidade de vida das pessoas (La Picirelli de Souza et al., 2021). Pois, segundo a Agência Nacional de Energia Elétrica - Aneel, hoje estão em operação no país cerca 560 termelétricas que utilizam biomassa. O bagaço de cana-de-açúcar, é o principal combustível de grande parcela dessas termelétricas. Outro resíduo que é muito utilizado atualmente são os relacionados a madeira, resíduos florestais, resíduos industriais de madeira na forma de cavacos ou serragem (ANEEL, 2018).

Tendo em vista os problemas abordados sobre a dependência da energia hidrelétrica no Brasil, o crescimento e desenvolvimento de outras fontes limpas e renováveis é de grande importância para o bom funcionamento do país. Com isso, esse trabalho propõe a utilização de resíduos sólidos urbanos como alternativa energética para a geração termoelétrica de energia. Para isso, os resíduos sólidos urbanos de poda de gramíneas do Distrito de Primavera, no município de Rosana-SP foram coletados, tratados e caracterizados por métodos gravimétricos e calorimétrico para avaliar a viabilidade de utilização com fonte de energia em processos de combustão direta. 


\section{Metodologia}

A metodologia utilizada para o desenvolvimento deste trabalho foi a pesquisa experimental como descrita por Pereira e seus colaboradores (Pereira et al., 2018). As etapas de pré-tratamento das amostras, análises de umidade, sólidos totais, extrativos aquosos e alcoólicos, bem como os inorgânicos estruturais e não estruturais foram baseadas nos Laboratory Analytical Procedures (LAPs) do National Renewable Energy Laboratory (NREL) (Hames et al., 2008; Sluiter, Hames, Ruiz, et al., 2008; Sluiter, Hames, Hyman, et al., 2008; Sluiter, Ruiz, et al., 2008).

\subsection{Pré-tratamento das amostras}

Para a caracterização dos resíduos urbanos sólidos de poda de gramíneas (RSPG) foram necessários os processos de pré-tratamento das amostras, os quais são otimizados com relação ao tipo de matriz sublenhosas, este processo se dá como necessário uma vez que esta matriz apresenta materiais indesejados (Silva et al., 2021). Esse tipo de amostra é uma biomassa sujeita à poluição e contaminação com diversos outros resíduos sólidos urbanos, ou ainda compostos não estruturais, que geram alterações consideráveis nos resultados, impossibilitando a obtenção de resultados precisos. Portanto, foram realizadas as etapas de coleta, lavagem, secagem e fracionamento baseados no procedimento do NREL (Hames et al., 2008).

A coleta é uma etapa direta, uma vez que por ser resíduo de poda, o material foi coletado em campo após o processo de poda. Foram coletadas amostras de RSPG no distrito de Primavera ( $22^{\circ} 34^{\prime} 48^{\prime \prime} \mathrm{S} ; 53^{\circ} 03^{\prime} 32^{\prime \prime} \mathrm{O}$ ) localizado no município de Rosana - SP (Figura 1).

Figura 1. Mapa do Distrito de Primavera (Rosana-SP) com os pontos de coleta das amostras em vermelho.

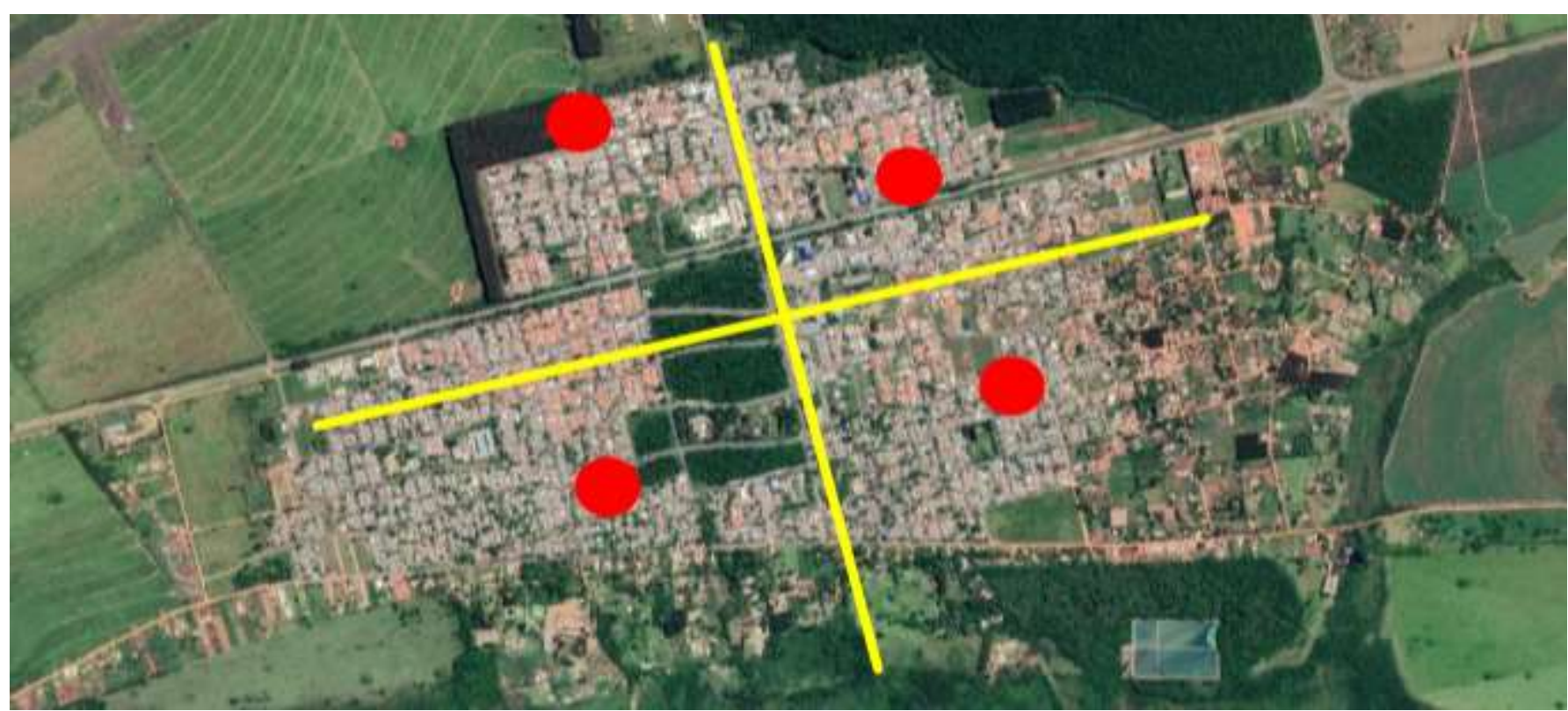

Fonte: Adaptado de (Google, 2021).

A Figura 1 mostra os pontos de coleta das amostras, as quais foram coletadas em quatro pontos diferentes com a finalidade de obtenção de resultados representativos.

Antes das lavagens foi realizado o procedimento de retirada de materiais não desejados, tais como raízes e folhas de árvores. Em seguida, as lavagens das amostras foram realizadas com água corrente para a eliminação de resíduos de terra. Após a lavagem, as amostram de RSPG foram submetidas ao processo de secagem ao ar (Hames et al., 2008) durante 24 h, ou até a umidade abaixo de $10 \%$, visando retirar a água adsorvida e auxiliar o processo de fracionamento. Após a secagem as amostras, cerca de $200 \mathrm{~g}$ de cada, foram fracionadas com um processador de facas e peneiradas em uma peneira 
granulométrica com abertura de $2 \mathrm{~mm}$. Após o processo de peneiração $50 \mathrm{~g}$ de cada amostra foram adicionadas em um recipiente cilíndrico e misturadas mecanicamente, obtendo assim amostras homogêneas e hábeis aos processos de caracterização.

\subsection{Umidade e sólidos totais}

Os testes de umidade e sólidos totais foram realizados em estufa convencional (Sluiter, Hames, Hyman, et al., 2008) e validados com um analisador de umidade modelo MOC63u da SHIMADZU. Primeiramente os cadinhos foram tarados, os quais foram aquecidos em estufa a $105 \pm 5^{\circ} \mathrm{C}$ por $12 \mathrm{~h}$. Após secos foram resfriados a temperatura ambiente em dessecador e pesados em balança analítica $\left( \pm 0.1 \mathrm{mg}\right.$ ). Sendo a massa do cadinho tarado denominada de $\mathrm{M}_{\text {beq }}$. Em seguida pesou-se cerca de $1000.0 \mathrm{mg}( \pm 0.1 \mathrm{mg})$ da amostra de RSPG $\left(\mathrm{M}_{\mathrm{pg}}\right)$, a massa foi adicionada nos cadinhos tarados $\left(\mathrm{M}_{\mathrm{beq}+\mathrm{pg}}\right)$ e transferidos para estufa, os quais foram mantidos por $12 \mathrm{~h}$ a $105 \pm 5^{\circ} \mathrm{C}$, após a eliminação da umidade este foi denominado de RSPG ODw $_{\text {(ODW }}$ - massa da amostra excluindo a umidade). Para os cálculos foram utilizadas as Equações 1 a 3.

$$
\begin{aligned}
& \mathrm{RSPG}_{\mathrm{ODW}}=\mathrm{M}_{\mathrm{beq}+\mathrm{Mpg}}-\mathrm{M}_{\mathrm{beq}} \\
& \% \text { Umidade }=1-\left(\mathrm{RSPG}_{\mathrm{ODW}} / \mathrm{M}_{\mathrm{pg}}\right) \times 100 \\
& \% \text { Sólidos Totais }=\left(\mathrm{RSPG}_{\mathrm{ODW}} / \mathrm{M}_{\mathrm{pg}}\right) \times 100
\end{aligned}
$$

\subsection{Extrativos aquosos e alcoólicos}

O processo de extração aquosa e com solvente (etanol) foi realizado de forma similar ao procedimento descrito na literatura (Sluiter, Ruiz, et al., 2008), com o objetivo de eliminar materiais não estruturais solúveis nas amostras de RSPG. O processo de extração aquosa e alcoólica foram realizados em um banho ultrassônico com no mínimo de três análises para cada amostra. Primeiro foi realizada a extração aquosa e depois a extração alcoólica e as etapas do procedimento estão descritas a seguir:

A - Separação de amostras homogêneas do RSPGodw;

B - Utilização de $100 \mathrm{~mL}$ de solvente (água deionizada ou etanol) para cada $1.00 \mathrm{~g}$ de amostra;

C - Adição da amostra de RSPG e o solvente em um Erlenmeyer;

D - Adição do Erlenmeyer no banho ultrassônico por 2 h;

$\mathrm{E}$ - Após as $2 \mathrm{~h}$, filtração em um funil de Büchner com papel de filtro quantitativo de filtragem rápida;

$\mathrm{F}$ - Secagem em estufa à $105^{\circ} \mathrm{C}$ por $12 \mathrm{~h}$ ou até atingir massa constante.

\subsection{Análise de cinzas}

Para obter valores pertinentes à quantidade de cinzas presentes no RSPU, foram utilizadas amostras RSPGodw livre de extrativos, ou seja, contendo apenas sólidos e livre de materiais não estruturais. Para realizar os testes, foram utilizados cadinhos de porcelana sem tampa em mufla modelo J200 da LUCADEMA, com pequenas modificações nas rampas de aquecimento, sendo que o procedimento foi similar ao Laboratory Analytical Procedure (LAP) do NREL para determinação de cinzas (Sluiter, Hames, Ruiz, et al., 2008). Os cálculos da porcentagem de cinzas foram realizados considerando as amostras

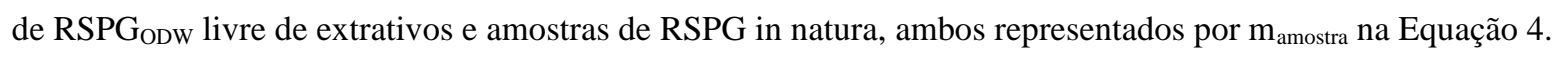

$\%$ Cinzas $=\left[\left(\mathrm{m}_{\text {cadinho+amostra }}-\mathrm{m}_{\text {cadinho }}\right) / \mathrm{m}_{\text {amostra }}\right] \times 100$ 


\subsection{Poder Calorífico Superior}

As determinações do poder calorífico superior (PCS) foram realizadas com uma bomba calorimétrica da marca IKA modelo C200 para as amostras de RSPGoDw livre de extrativos.

\section{Resultados e Discussão}

3.1 Análises de Umidade, Sólidos Totais, Extrativos, Inorgânicos Totais e Cinzas do RSPG.

A Tabela 1 mostra os resultados obtidos de umidade, sólidos totais, extrativos aquoso e alcoólico, inorgânicos estruturais e não-estruturais, inorgânicos totais e cinzas do RSPG.

Tabela 1. Valores de umidade, sólidos totais, extrativos aquoso e alcoólico, inorgânicos estruturais e não-estruturais, inorgânicos totais e cinzas para as amostras de RSPG.

\begin{tabular}{lc}
\hline Parâmetro & Valores em Porcentagem \\
\hline Umidade & $9,60 \pm 0,38 \%$ \\
Sólidos Totais & $90,40 \pm 0,38 \%$ \\
Extrativo Aquoso & $14,32 \pm 0,28 \%$ \\
Extrativo Alcoólico (etanol) & $6,65 \pm 0,40 \%$ \\
Cinzas (RSPGodw livre de extrativos) & $2,97 \pm 0,17 \%$ \\
Cinzas (RSPG in natura) & $4,23 \pm 0,05 \%$ \\
Inorgânicos estruturais & $2,97 \pm 0,17 \%$ \\
Inorgânicos não estruturais & $1,26 \pm 0,12 \%$ \\
Inorgânicos Totais & $4,23 \pm 0,05 \%$ \\
\hline
\end{tabular}

Fonte: Autores.

A Tabela 1 mostra os resultados das análises realizadas com os valores médios e de desvio padrão.

A determinação de umidade foi realizada nas amostras secas ao ar por $24 \mathrm{~h}$, foram pesadas em cadinhos de porcelana

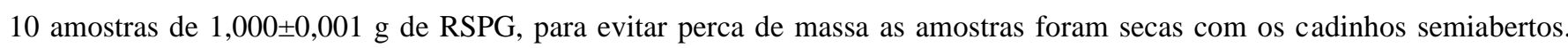
Após a secagem e pesagem, até obtenção de massa constante o valor em porcentagem da umidade foi calculada em relação ao

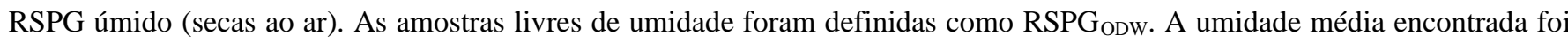

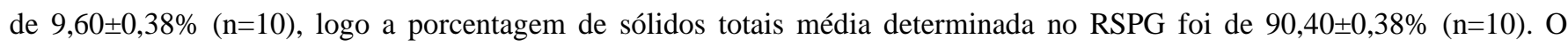
analisador de umidade (MOC63u - SHIMADZU) foi utilizado, como forma de validar os resultados encontrados pelo método

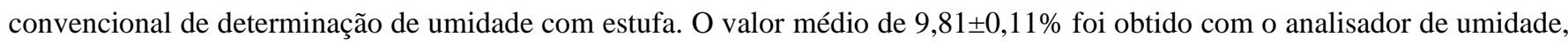
portanto observou que os dois métodos apresentaram resultados convergentes.

Os materiais inorgânicos e orgânicos estão presentes em amostras de biomassa (Alonso-Riaño et al., 2020; Du et al., 2020). Cinza estrutural é um material inorgânico que está ligado na estrutura física da biomassa, enquanto a cinza extraível é o material inorgânico que pode ser removido por meio de lavagem ou de extração. Portanto as cinzas foram quantificadas tanto na amostra in natura (pré-extração) e na amostra após processos de extração aquosa e etanólica. Sendo que os inorgânicos estruturais foram determinados pelas cinzas nas amostras livre de extrativos e os inorgânicos não estruturais determinados pela diferença das cinzas totais com as cinzas após extrações aquosa e etanólica.

A extração aquosa é responsável pela retirada de materiais hidrofílicos não-estruturais das amostras, já a extração etanólica é necessária para a extração de ceras e lipídeos (Sluiter, Ruiz, et al., 2008). Os testes de extração foram realizados em 
diferentes tempos (1, 2, 3 e $4 \mathrm{~h})$ e não foi observada uma diferença significativa de extrativos entre os tempos de 2 e $4 \mathrm{~h}$, ou seja, a porcentagem de extrativos observados em $2 \mathrm{~h}$ foi similar ao tempo de 3 e $4 \mathrm{~h}$, com variação de em torno de 2 a $3 \%$ nos valores de extrativos. Portanto, o tempo de $2 \mathrm{~h}$ foi escolhido para fazer a remoção dos extrativos.

A quantidade dos extrativos aquosos $(14,32 \pm 0,28 \%)$ encontrados foram cerca de duas vezes maior do que os extrativos etanólicos $(6,65 \pm 0,40 \%)$ indicando que nas amostras havia uma quantidade maior de compostos inorgânicos e orgânicos não-estruturais fisicamente ligados à estrutura do bagaço do que compostos orgânicos obtidos pela extração etanólica, como as ceras e lipídeos.

Os testes realizados para determinação de cinzas, foram realizados em duas etapas, sendo a primeira, realizada com amostras in natura (RSPG in natura) e a segunda etapa com a biomassa proveniente após os extrativos etanólicos (RSPGoDw livre de extrativos). Ao realizar o teste para determinação de cinzas com ambas as amostras, in natura e pós extrativos, é possível realizar uma análise matemática entre ambos os resultados, o que por fim é caracterizado por Teste de Inorgânicos estruturais e não estruturais. Ao realizar a comparação das cinzas provenientes de uma biomassa in natura com as da biomassa pós-tratamentos extrativos laboratoriais, foi possível determinar uma porcentagem, que determina justamente uma parcela de impurezas inorgânicas não intrínsecas ao recurso sublenhoso, atribuindo esta diferença como extrativos não estruturais.

Ao analisar o presente valor percentual de inorgânicos estruturais de $2,97 \pm 0,17 \%$ obtido pela análise de cinzas no

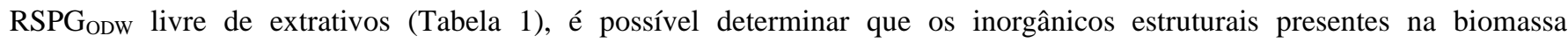
representam apenas uma mínima fração da massa como um todo. Este valor embora mínimo, é importante ser levado em consideração, uma vez que representa uma porcentagem da massa total da qual não é possível extrair energia, apesar de ser meramente impureza, é ideal tomar o conhecimento dele, uma vez que está presente em toda massa considerada desta biomassa.

A variação no teor de cinzas representada pela diferença entre o teor de cinzas do RSPG in natura e o teor das cinzas do RSPGodw livre de extrativos representam a taxa de inorgânicos não estruturais no composto, ou seja, 1,26 $\pm 0,12 \%$ são os considerados inorgânicos não estruturais, os quais são responsáveis por 29,8\% das cinzas totais do RSPG in natura e seco ao ar. Apesar de compor uma pequena taxa do recurso como um todo, estes compostos denominados por inorgânicos estruturais e não estruturais são um fator importante que implica na formação das cinzas durante o processo de queima direta.

\subsection{Análises do Poder Calorífico Superior do RSPG.}

Com a separação de $10,0 \mathrm{~g}$ da amostra referente às amostras pós extrativos aquosos, foram realizadas as devidas amostras em cadinhos e inseridos na bomba calorimétrica. O valor médio do PCS foi de 17,59 $\pm 0,43 \mathrm{MJ} \mathrm{kg}^{-1}$ (Tabela 2).

Tabela 2. Comparação do PCS para diferentes biomassas.

\begin{tabular}{lcc}
\hline Resíduos & PCS $\left(\mathbf{M J ~ k g}^{-1}\right)$ & Ref. \\
\hline Eucalipto & 18,95 & (Dias et al., 2021) \\
Bagaço de cana-de-açúcar & 18,16 & (Coelho et al., 2019) \\
Palha de cana-de-açúcar & 17,38 & (Canettieri et al., 2018) \\
Poda de jardim & 15,13 & (Vallejo et al., 2020) \\
Talhos de milho & 16,54 & (Galhano dos Santos et al., 2018) \\
Casca de arroz & 16,78 & (Galhano dos Santos et al., 2018) \\
Resíduo Sólido Urbano de Poda de Gramíneas & 17,59 & (Esse Trabalho) \\
\hline
\end{tabular}

Fonte: Autores. 
O valor de PCS obtido representa o potencial energético químico intrínseco à biomassa, ou ainda, a capacidade da matéria orgânica em oferecer energia térmica (Enes et al., 2019). Compreendendo que esta energia se encontra na forma química, pode ser aproveitada através da queima direta. Ao considerar o poder calorífico superior, pode-se considerar que o RSPG são passíveis de serem adotados como alternativa para geração de energia por queima direta, uma vez que ao analisar a Tabela 2, é possível observar que o PCS obtido é semelhante a diferentes resíduos já aplicados em processos industriais e descritos na literatura. O RSPG apresentou valores de PCS semelhantes aos de substâncias como o bagaço de cana-de-açúcar (Coelho et al., 2019) e ao palhiço da cana-de-açúcar (Canettieri et al., 2018), o que mostra sua viabilidade energética para a aplicação como combustível sólido para a queima direta.

\section{Conclusão}

Conclui-se por fim que o resíduo sólido urbano de poda de gramíneas apresenta características para o aproveitamento energético, tendo uma composição semelhante a resíduos já inseridos no mercado e que apresentam um valor comercial. O RSPG apresentou ser alternativa como combustível sólido ao apresentar um potencial energético, ou seja, um PCS de 17,59 $\pm 0,43 \mathrm{MJ} \mathrm{kg}^{-1}$ que é similar a outros resíduos sólidos, o seu diferencial é justamente por ser um resíduo não aproveitado e que pode ser inserido na matriz, sendo utilizado para aplicações de cogeração de energia, de modo semelhante àquelas utilizadas por outros resíduos, como o bagaço de cana-de-açúcar. Este resíduo mostrou ser uma saída viável e alternativa frente a necessidade energética em desenvolver novos recursos energéticos, uma vez que este, atualmente, é considerado apenas como resíduo, sendo descartado nos aterros sanitários como é o caso do município de Rosana.

Para trabalhos futuros será realizada a avaliação do potencial energético dos produtos de resíduos de poda urbana obtidos pelos processos de pirólise e gaseificação, tais como o bio-óleo, carvão mineral e o gás combustível, que são produtos com elevados valores de poder calorífico. Outro ponto a ser estudado é a densificação energética dessa biomassa através da produção de pellets ou briquetes e avaliação dos parâmetros físico-químicos.

\section{Referências}

Alonso-Riaño, P., Sanz Diez, M. T., Blanco, B., Beltrán, S., Trigueros, E., \& Benito-Román, O. (2020). Water Ultrasound-Assisted Extraction of Polyphenol Compounds from Brewer's Spent Grain: Kinetic Study, Extract Characterization, and Concentration. Antioxidants, 9(3), 265. https://doi.org/10.3390/antiox9030265.

ANEEL. (2018). Relatório de desempenho das usinas termoelétricas despachadas centralizadamente - TIPOS I E IIA (Issue 12). Agência Nacional De Energia Elétrica - ANEEL.

Canettieri, E. V., da Silva, V. P., Neto, T. G. S., Hernández-Pérez, A. F., da Silva, D. D. V., Dussán, K. J., das Graças Almeida Felipe, M., \& de Carvalho, J. A. (2018). Physicochemical and thermal characteristics of sugarcane straw and its cellulignin. Journal of the Brazilian Society of Mechanical Sciences and Engineering, 40(9), 416. https://doi.org/10.1007/s40430-018-1331-1.

Coelho, R. D., Lizcano, J. V., da Silva Barros, T. H., da Silva Barbosa, F., Leal, D. P. V., da Costa Santos, L., Ribeiro, N. L., Júnior, E. F. F., \& Martin, D. L. (2019). Effect of water stress on renewable energy from sugarcane biomass. Renewable and Sustainable Energy Reviews, 103 (April 2018 ), 399-407. https://doi.org/10.1016/j.rser.2018.12.025.

de Sousa, M. H., da Silva, A. S. F., Correia, R. C., Leite, N. P., Bueno, C. E. G., dos Santos Pinheiro, R. L., de Santana, J. S., da Silva, J. L., Sales, A. T., de Souza, C. C., da Silva Aquino, K. A., de Souza, R. B., Pinheiro, I. O., Henríquez, J. R., Schuler, A. R. P., de Sá Barretto Sampaio, E. V., Dutra, E. D., \& Menezes, R. S. C. (2021). Valorizing municipal organic waste to produce biodiesel, biogas, organic fertilizer, and value-added chemicals: an integrated biorefinery approach. Biomass Conversion and Biorefinery. https://doi.org/10.1007/s13399-020-01252-5.

Dias, A. F., Arthuso, H., Lana, A. Q., Andrade, C. R., Brito, J. O., \& Júnior, G. B. (2021). Addition of Eucalyptus sp wood to urban wood waste as a strategy for energetic use. Scientia Forestalis/Forest Sciences, 49(129), 1-11. https://doi.org/10.18671/SCIFOR.V49N129.05.

Du, L., Arauzo, P. J., Meza Zavala, M. F., Cao, Z., Olszewski, M. P., \& Kruse, A. (2020). Towards the properties of different biomass-derived proteins via various extraction methods. Molecules, 25(3), 488. https://doi.org/10.3390/molecules25030488.

Empresa de Pesquisa Energética. (2021). Balanço Energético Nacional. Empresa de Pesquisa Energética. https://www.epe.gov.br/pt/publicacoes-dadosabertos/publicacoes/balanco-energetico-nacional-2021.

Enes, T., Aranha, J., Fonseca, T., Lopes, D., Alves, A., \& Lousada, J. (2019). Thermal properties of residual agroforestry biomass of northern Portugal. Energies, 12(8), 1418. https://doi.org/10.3390/en12081418. 
Evaristo, R. B. W., Viana, N. A., Guimarães, M. G., do Vale, A. T., de Macedo, J. L., \& Ghesti, G. F. (2020). Evaluation of waste biomass gasification for local community development in central region of Brazil. Biomass Conversion and Biorefinery. https://doi.org/10.1007/s13399-020-00821-y.

Galhano dos Santos, R., Bordado, J. C., \& Mateus, M. M. (2018). Estimation of HHV of lignocellulosic biomass towards hierarchical cluster analysis by Euclidean's distance method. Fuel, 221(February), 72-77. https://doi.org/10.1016/j.fuel.2018.02.092.

Gomes, L., Miranda, H. S., Soares-Filho, B., Rodrigues, L., Oliveira, U., \& Bustamante, M. M. C. (2020). Responses of Plant Biomass in the Brazilian Savanna to Frequent Fires. Frontiers in Forests and Global Change, 3(November), 1-11. https://doi.org/10.3389/ffgc.2020.507710.

Google. (2021). Mapa do Distrito de Primavera, Rosana-SP. Google Maps. https://www.google.com.br/maps/@-22.5359732,$52.9514191,2755 \mathrm{~m} /$ data $=! 3 \mathrm{~m} 1 ! 1 \mathrm{e} 3$ ?hl=pt-BR.

Haidar, D. (2021). Maior hidrelétrica de SP atinge "0\%" do reservatório e chega ao volume morto. https://www.metropoles.com/brasil/maior-hidreletrica-desp-atinge-0-do-reservatorio-e-chega-ao-volume-morto.

Hames, B., Ruiz, R., Scarlata, C., Sluiter, A., Sluiter, J., \& Templeton, D. (2008). Preparation of Samples for Compositional Analysis: Laboratory Analytical Procedure (LAP) (TP-510-42620). National Renewable Energy Laboratory. https://www.nrel.gov/bioenergy/biomass-compositional-analysis.html.

Hiloidhari, M., \& Baruah, D. C. (2011). Crop residue biomass for decentralized electrical power generation in rural areas (part 1): Investigation of spatial availability. Renewable and Sustainable Energy Reviews, 15(4), 1885-1892. https://doi.org/10.1016/j.rser.2010.12.010.

La Picirelli de Souza, L., Rajabi Hamedani, S., Silva Lora, E. E., Escobar Palacio, J. C., Comodi, G., Villarini, M., \& Colantoni, A. (2021). Theoretical and technical assessment of agroforestry residue potential for electricity generation in Brazil towards 2050. Energy Reports, 7, $2574-2587$. https://doi.org/10.1016/j.egyr.2021.04.026.

Miranda, R. B. de, Scarpinella, G. D., \& Mauad, F. F. (2013). Influência do assoreamento na capacidade de armazenamento do Reservatório da usina hidrelétrica de Três Irmãos (SP/BRASIL). Revista Recursos Hídricos, 34(2), 69-79. https://doi.org/10.5894/rh34n2-6.

Pereira, A. S., Shitsuka, D. M., Parreira, F. J., \& Shitsuka, R. (2018). Metodologia da Pesquisa Científica. UFSM. https://repositorio.ufsm.br/bitstream/h andle/1/15824/Lic_Computacao_Metodologia-Pesquisa-Cientifica.pdf?sequence=1

Silva, J. B. S. da, Torquato, L. D. M., Crnkovic, P. M., \& Cruz, G. (2021). Investigation Of The Urban Pruning Wastes As Biofuels And Possible Utilization In Thermal Systems / Investigação Dos Resíduos Da Poda Urbana Como Biocombustíveis E Possível Utilização Em Sistemas Térmicos. Brazilian Journal of Development, 7(3), 24730-24750. https://doi.org/10.34117/bjdv7n3-265.

Sluiter, A., Hames, B., Hyman, D., Payne, C., Ruiz, R., Scarlata, C., Sluiter, J., Templeton, D., \& Wolfe, J. (2008). Determination of total solids in biomass and total dissolved solids in liquid process samples: Laboratory Analytical Procedure (LAP) (TP-510-42621). National Renewable Energy Laboratory. https://www.nrel.gov/bioenergy/biomass-compositional-analysis.html.

Sluiter, A., Hames, B., Ruiz, R., Scarlata, C., Sluiter, J., \& Templeton, D. (2008). Determination of Ash in Biomass: Laboratory Analytical Procedure (LAP) (TP-510-42622). National Renewable Energy Laboratory. https://www.nrel.gov/bioenergy/biomass-compositional-analysis.html.

Sluiter, A., Ruiz, R., Scarlata, C., Sluiter, J., \& Templeton, D. (2008). Determination of Extractives in Biomass: Laboratory Analytical Procedure (LAP) (TP510-42619). National Renewable Energy Laboratory. https://www.nrel.gov/bioenergy/biomass-compositional-analysis.html.

Vallejo, F., Díaz-Robles, L., Vega, R., Cubillos, F., Espinoza, A. P., Pinilla, F., \& Pino-Cortés, E. (2020). An experimental study for municipal organic waste and sludge treated by hydrothermal carbonization. Chemical Engineering Transactions, 81(August), 355-360. https://doi.org/10.3303/CET2081060. 